\title{
The Structure of Reflective Function of Higher Dimensional Differential System
}

\author{
Zhengxin Zhou \\ Department of Mathematics, Yangzhou University, Yangzhou, China \\ E-mail: zhengxinzhou@hotmail.com \\ Received March 16, 2010; revised April 18, 2010; accepted April 29, 2010
}

\begin{abstract}
In this article, we discuss the structure of reflective function of the higher dimensional differential systems and apply the results to study the existence of periodic solutions of these systems.
\end{abstract}

Keywords: Reflecting Function, Periodic Solution, Higher Dimensional System

\section{Introduction}

As we know, to study the property of the solutions of differential system

$$
x^{\prime}=X(t, x)
$$

is very important not only for the theory of ordinary differential equation but also for practical reasons. If $X(t+2 \omega, x)=X(t, x) \quad(\omega$ is a positive constant $)$, to study the solutions' behavior of (1), we could use, as introduced in [1], the Poincare mapping. But it is very difficult to find the Poincare mapping for many systems which cannot be integrated in quadratures. In the 1980's the Russian mathematician Mironenko [2] first established the theory of reflective functions (RF). Since then a quite new method to study (1) has been found.

In the present section, we introduce the concept of the reflective function, which will be used throughout the rest of this article.

Now consider the system (1) with a continuously differentiable right-hand side and with a general solution $\psi\left(t ; t_{0}, x_{0}\right)$. For each such system, the reflective function (RF) of (1) is defined as $F(t, x(t))=\psi(-t ; t, x)$. Then for any solution $x(t)$ of (1), we have $F(t, x(t))=$ $: x(-t)$. If system (1) is $2 \omega$-periodic with respect to $t$, and $F(t, x)$ is its RF, then $F(-\omega, x)=\psi(\omega ;-\omega, x)$ is the Poincare [1-2] mapping of (1) over the period $[-\omega, \omega]$. So, for any solution $x(t)$ of (1) defined on $[-\omega, \omega]$, it will be $2 \omega$--periodic if and only if $x(-\omega)$ is a fixed point of the Poincare mapping $T(x)=F(-\omega, x)$. A function $F(t, x)$ is a reflective function of system (1) if and only if it is a solution of the partial differential equation (called a basic relation, BR)

$$
F_{t}^{\prime}+F_{x}^{\prime} X(t, x)+X(-t, x)=0
$$

with the initial condition $F(0, x)=x$. It implies that for non-integrable periodic systems we also can find out its Poincare mapping. If, for example,

$$
X(t, x)+X(-t, x)=0 \text {, then } T(x)=x .
$$

If $F(t, x)$ is the RF of (1), then it is also the RF of the system

$$
x^{\prime}=X(t, x)+F_{x}^{-1} R(t, x)-R(-t, F(t, x)),
$$

where $R(t, x)$ is an arbitrary vector function such that the solutions of the above systems are uniquely determined by their initial conditions. Therefore, all these $2 \omega$-periodic systems have a common Poincare mapping over the period $\mathrm{f} \omega, \omega]$, and the behavior of the periodic solutions of these systems are the same.

To find out the reflective function is very important for studying the qualitative behavior of solutions of differential systems. The literatures [5-8] have discussed the structure of the reflective function of some second order quadric systems and linear systems and obtained many good results.

Now, we consider the higher dimensional polynomial differential system

$$
\left\{\begin{array}{c}
x^{\prime}=p_{1}+p_{2} y+p_{3} z=P(t, x, y, z) \\
y^{\prime}=q_{1}+q_{2} y+q_{3} z+q_{4} y^{2}+q_{5} y z+q_{6} z^{2}=Q(t, x, y, z)(3) \\
z^{\prime}=r_{1}+r_{2} y+r_{3} z+r_{4} y^{2}+r_{5} y z+r_{6} z^{2}=R(t, x, y, z)
\end{array}\right.
$$

where

$$
\begin{aligned}
p_{i} & =p_{i}(t, x), q_{j}=q_{j}(t, x), r_{j}=r_{j}(t, x) \\
(i & =1,2,3 ; j=1,2, \ldots, 6)
\end{aligned}
$$

are continuously differentiable functions in $\mathrm{R}^{2}$, and $p_{2}^{2}+p_{3}^{2} \neq 0$ (in some deleted neighborhood of $t=0$ 
and $|t|$ being small enough, $p_{2}^{2}+p_{3}^{2} \neq 0$ is different from zero), and there exists a unique solution for the initial value problem of (3). And suppose that $F(t, x, y, z)=\left(F_{1}(t, x, y, z), F_{2}(t, x, y, z), F_{3}(t, x, y, z)\right)^{T}$ is the RF of (3).

In this paper, we will discuss the structure of $F_{i}(t, x, y, z)(i=2,3)$ when $F_{1}(t, x, y, z)=f(t, x)$. At the same time, we obtain the good results that $F_{i}(t, x, y, z)=f_{i 1}(t, x)+f_{i 2}(t, x) y+f_{i 3}(t, x) z \quad(i=2,3) \quad$.

The obtained results are used for research of problems of existence of periodic solution of the system (3) and establish the sufficient conditions under which the first component of the solution of (3) is even function.

In the following, we will denote

$\bar{p}_{i}=p_{i}(-t, x) ; \bar{q}_{j}=q_{j}(-t, x) ; \bar{r}_{j}=r_{j}(t, x) ; F_{i}=F_{i}(t, x, y, z)$, $i=1,2,3, j=1,2, \ldots, 6$. The notation $p_{i}(t, x) \neq 0$ means that, in some deleted neighborhood of $t=0$ and $|t|$ being small enough, $p_{i}(t, x)$ is different from zero,

$$
D A=\frac{\partial A}{\partial t}+\frac{\partial A}{\partial x} P(t, x, y, z)+\frac{\partial A}{\partial y} Q(t, x, y, z)+\frac{\partial A}{\partial z} R(t, x, y, z) .
$$

\section{Main Results}

Without loss of generality, we suppose that

$f(t, x)=x$. Otherwise, we take the transformation $\xi=f(t, x), \eta=y, \zeta=z$.

Now, let's consider the system (3)

Lemma 1. For the system (3), suppose that $F_{1}=x$. Then

$$
p_{i}(t, x)=0, i=1,2,3 .
$$

Proof. Using the relation (2), we get

$$
P(t, x, y, z)+P\left(-t, x, F_{2}, F_{3}\right)=0,
$$

i.e.

$$
p_{1}+\bar{p}_{1}+p_{2} y+p_{3} z+\bar{p}_{2} F_{2}+\bar{p}_{3} F_{3}=0 .
$$

Putting $t=0$, we get

$$
p_{1}(0, x)+p_{2}(0, x) y+p_{3}(0, x) z \equiv 0, \forall x, y, z .
$$

It implies that the relation (4) is valid.

In the following discussion, we always assume (4) holds without further mention.

Case 1. $p_{3} \neq 0$.

From (5), we get

where

$$
F_{3}=\lambda_{1}+\lambda_{2} F_{2}
$$

$$
\begin{aligned}
& \lambda_{1}=-\frac{p_{1}+\bar{p}_{1}}{\bar{p}_{3}}-\frac{p_{2}}{\bar{p}_{3}} y-\frac{p_{3}}{\bar{p}_{3}} z=\lambda_{11}+\lambda_{12} y+\lambda_{13} z, \\
& \lambda_{2}=-\frac{\bar{p}_{2}}{\bar{p}_{3}} .
\end{aligned}
$$

Differentiating relation (6) respect to $t$ implies where

$$
A_{0}+A_{1} F_{2}+A_{2} F_{2}^{2}=0,
$$

$$
\begin{aligned}
& A_{0}=D \lambda_{1}-\lambda_{2} \bar{q}_{1}+\bar{r}_{1}+\lambda_{1}\left(\bar{r}_{3}-\lambda_{2} \bar{q}_{3}\right)+\lambda_{1}^{2}\left(\bar{r}_{6}-\lambda_{2} \bar{q}_{6}\right) \\
& =a_{01}+a_{02} y+a_{03} z+a_{04} y^{2}+a_{05} y z+a_{06} z^{2} ; \\
& A_{1}=D \lambda_{2}-\lambda_{2} \bar{q}_{2}+\bar{r}_{2}+\lambda_{2}\left(\bar{r}_{3}-\lambda_{2} \bar{q}_{3}\right)+\lambda_{1}\left(\bar{r}_{5}-\lambda_{2} \bar{q}_{5}\right) ; \\
& +2 \lambda_{1} \lambda_{2}\left(\bar{r}_{6}-\lambda_{2} \bar{q}_{6}\right)=a_{11}+a_{12} y+a_{13} z ; \\
& A_{2}=A_{2}(t, x)=-\lambda_{2} \bar{q}_{4}+\bar{r}_{4}+\lambda_{2}\left(\bar{r}_{5}-\lambda_{2} \bar{q}_{5}\right)+\lambda_{2}^{2}\left(\bar{r}_{6}-\lambda_{2} \bar{q}_{6}\right),
\end{aligned}
$$

In which

$$
\begin{aligned}
a_{01}= & \lambda_{11 t}^{\prime}+\lambda_{11 x}^{\prime} p_{1}+\lambda_{12} q_{1}+\lambda_{13} r_{1}+\bar{r}_{1}-\lambda_{2} \bar{q}_{1}+ \\
& +\lambda_{11}\left(\bar{r}_{3}-\lambda_{2} \bar{q}_{3}\right)+\left(\bar{r}_{6}-\lambda_{2} \bar{q}_{6}\right) \lambda_{11}^{2} ; \\
a_{02}= & \lambda_{12 t}^{\prime}+\lambda_{12 x}^{\prime} p_{2}+\lambda_{12 x}^{\prime} p_{1}+\lambda_{12} q_{2}+\lambda_{13} r_{2}+ \\
& +\lambda_{12}\left(\bar{r}_{3}-\lambda_{2} \bar{q}_{3}\right)+2\left(\bar{r}_{6}-\lambda_{2} \bar{q}_{6}\right) \lambda_{11} \lambda_{12} ; \\
a_{03}= & \lambda_{13 t}^{\prime}+\lambda_{1 x}^{\prime} p_{3}+\lambda_{13 x}^{\prime} p_{3}+\lambda_{12} q_{3}+\lambda_{13} r_{3}+ \\
& +\lambda_{13}\left(\bar{r}_{3}-\lambda_{2} \bar{q}_{3}\right)+2\left(\bar{r}_{6}-\lambda_{2} \bar{q}_{6}\right) \lambda_{11} \lambda_{13} ; \\
a_{04}= & \lambda_{12 x}^{\prime} p_{2}+\lambda_{12} q_{4}+\lambda_{13} r_{4}+\left(\bar{r}_{6}-\lambda_{2} \bar{q}_{6}\right) \lambda_{12}^{2} ; \\
a_{05}= & \lambda_{12 x}^{\prime} p_{3}+\lambda_{13 x}^{\prime} p_{2}+\lambda_{12} q_{5}+\lambda_{13} r_{5}+2\left(\bar{r}_{6}-\lambda_{2} \bar{q}_{6}\right) \lambda_{12} \lambda_{13} ; \\
a_{06}= & \lambda_{13 x}^{\prime} p_{3}+\lambda_{12} q_{6}+\lambda_{13} r_{6}+\left(\bar{r}_{6}-\lambda_{2} \bar{q}_{6}\right) \lambda_{13}^{2} ; \\
a_{11}= & \lambda_{2 t}^{\prime}+\lambda_{2 x}^{\prime} p_{1}+\bar{r}_{2}-\lambda_{2} \bar{q}_{2}+\lambda_{2}\left(\bar{r}_{3}-\lambda_{2} \bar{q}_{3}\right)+ \\
& +\lambda_{11}\left(\bar{r}_{5}-\lambda_{2} \bar{q}_{5}\right)+2\left(\bar{r}_{6}-\lambda_{2} \bar{q}_{6}\right) \lambda_{2} \lambda_{11} ; \\
a_{12}= & \lambda_{2 x}^{\prime} p_{2}+\lambda_{12}\left(\bar{r}_{5}-\lambda_{2} \bar{q}_{5}\right)+2\left(\bar{r}_{6}-\lambda_{2} \bar{q}_{6}\right) \lambda_{2} \lambda_{12} ; \\
a_{13}= & \lambda_{2 x}^{\prime} p_{3}+\lambda_{13}\left(\bar{r}_{5}-\lambda_{2} \bar{q}_{5}\right)+2\left(\bar{r}_{6}-\lambda_{2} \bar{q}_{6}\right) \lambda_{2} \lambda_{13} ; \\
\lambda_{11}= & -\frac{p_{1}+\bar{p}_{1}}{\bar{p}_{3}}, \lambda_{12}=-\frac{p_{2}}{\bar{p}_{3}}, \lambda_{13}=-\frac{p_{3}}{\bar{p}_{3}} .
\end{aligned}
$$

Lemma 2.Let $F_{1}=x, A_{2} \neq 0$ and $\lim _{t \rightarrow 0} \frac{a_{0 i}}{A_{2}}(i=1,2, \ldots, 6)$

exist. Then $\lim _{t \rightarrow 0} \frac{a_{0 j}}{A_{2}}=0 \quad(j=1,3,6), \quad \lim _{t \rightarrow 0} \frac{a_{02}+a_{11}}{A_{2}}=0$,

$$
\lim _{t \rightarrow 0} \frac{a_{04}+a_{12}}{A_{2}}=-1, \lim _{t \rightarrow 0} \frac{a_{05}+a_{13}}{A_{2}}=0 .
$$

Proof. Using the relation (7), we have

$\lim _{t \rightarrow 0} \frac{a_{01}+a_{02} y+a_{03} z+a_{04} y^{2}+a_{05} y z+a_{06} z^{2}}{A_{2}}+$

$\lim _{t \rightarrow 0} \frac{a_{11}+a_{12} y+a_{13} z}{A_{2}} F_{2}+\lim _{t \rightarrow 0} F_{2}^{2}=0$,

As $F_{2}(0, x, y, z)=y$, it follows that the results of Lemma 2 are true.

Theorem 1. Let the conditions of Lemma 1 and Lemma 2 satisfy and $\lim _{t \rightarrow 0} \frac{a_{12}}{A_{2}}+2>0$. Then

$$
F_{i}=f_{i 1}(t, x)+f_{i 2}(t, x) y+f_{i 3}(t, x) z \quad(i=2,3) .
$$


Proof. AS $A_{2} \neq 0$. From (7), it follows

$$
F_{2}^{2}=-\frac{A_{0}+A_{1} F_{2}}{A_{2}}, F_{2}^{3}=\frac{A_{1} A_{0}}{A_{2}^{2}}+\frac{A_{1}^{2}-A_{0} A_{2}}{A_{2}^{2}} F_{2}
$$

Differentiating relation (7) respect to $t$ implies

$$
\begin{aligned}
& D A_{0}+D A_{1} F_{2}+D A_{2} F_{2}^{2}-A_{1} Q\left(-t, x, F_{2}, F_{3}\right) \\
& -2 A_{2} Q\left(-t, x, F_{2}, F_{3}\right) F_{2}=0 .
\end{aligned}
$$

Substituting (6) into the above, we get

$$
B_{0}+B_{1} F_{2}+B_{3} F_{2}^{2}+B_{4} F_{2}^{3}=0,
$$

where

$$
\begin{aligned}
& B_{0}=D A_{0}-A_{1} \mu_{1}, \quad B_{1}=D A_{1}-A_{1} \mu_{2}-2 A_{2} \mu_{1}, \\
& B_{2}=D A_{2}-A_{1} \mu_{3}-2 A_{2} \mu_{2}, \quad B_{4}=-2 A_{2} \mu_{3},
\end{aligned}
$$

in which

$$
\begin{aligned}
& \mu_{1}=\bar{q}_{1}+\bar{q}_{3} \lambda_{1}+\bar{q}_{6} \lambda_{1}^{2}, \mu_{2}=\bar{q}_{2}+\bar{q}_{3} \lambda_{2}+\bar{q}_{5} \lambda_{1}+2 \bar{q}_{6} \lambda_{2} \lambda_{1}, \\
& \mu_{3}=\bar{q}_{4}+\bar{q}_{5} \lambda_{2}+\bar{q}_{6} \lambda_{2}^{2} .
\end{aligned}
$$

Substituting (8) into (9), we have

$$
C_{0}+C_{1} F_{2}=0 \text {, }
$$

where

$$
\begin{aligned}
& C_{0}=B_{0}-B_{2} \frac{A_{0}}{A_{2}}+B_{3} \frac{A_{1} A_{0}}{A_{2}^{2}}, \\
& C_{1}=B_{1}-B_{2} \frac{A_{1}}{A_{2}}+B_{3} \frac{A_{1}^{2}-A_{0} A_{2}}{A_{2}^{2}} .
\end{aligned}
$$

$1^{*}$. If $C_{1} \equiv 0$, from (10) follows $C_{0} \equiv 0$. By simple computation, we obtain

$$
\begin{gathered}
D \frac{A_{0}}{A_{2}}=\mu_{1} \frac{A_{1}}{A_{2}}-2 \mu_{2} \frac{A_{0}}{A_{2}}+\mu_{3} \frac{A_{0} A_{1}}{A_{2}{ }^{2}}, \\
D \frac{A_{1}}{A_{2}}=2 \mu_{1}-\mu_{2} \frac{A_{1}}{A_{2}}+\mu_{3}\left(\frac{A_{1}^{2}}{A_{2}^{2}}-2 \frac{A_{0}}{A_{2}}\right) .
\end{gathered}
$$

Let $\Delta=\left(\frac{A_{1}}{A_{2}}\right)^{2}-4 \frac{A_{0}}{A_{2}}$. Using (11) (12) we get

$$
D \Delta=2\left(\mu_{3} \frac{A_{1}}{A_{2}}-\mu_{2}\right) \Delta .
$$

Since

$$
\begin{aligned}
\Delta= & \left(\frac{A_{1}}{A_{2}}\right)^{2}-4 \frac{A_{0}}{A_{2}}=\frac{1}{A_{2}^{2}}\left(\left(a_{11}+a_{12} y+a_{13} z\right)^{2}-\right. \\
& \left.4 A_{2}\left(a_{01}+a_{02} y+a_{03} z+a_{04} y^{2}+a_{05} y z+a_{06} z^{2}\right)\right) \\
= & d_{1}+d_{2} y+d_{3} z+d_{4} y^{2}+d_{5} y z+d_{6} z^{2} \\
= & d_{4}\left(y+\frac{d_{5}}{2 d_{4}} z+\frac{d_{2}}{2 d_{4}}\right)^{2}+W,
\end{aligned}
$$

where

$$
\begin{gathered}
W=\frac{1}{4 d_{4}}\left[\left(4 d_{4} d_{6}-d_{5}^{2}\right) z^{2}+2\left(2 d_{3} d_{4}-d_{2} d_{5}\right) z+4 d_{1} d_{4}-d_{2}^{2}\right], \\
d_{1}=\frac{1}{A_{2}^{2}}\left(a_{11}^{2}-4 A_{2} a_{01}\right), d_{2}=\frac{1}{A_{2}^{2}}\left(2 a_{11} a_{12}-4 A_{2} a_{02}\right), \\
d_{3}=\frac{1}{A_{2}^{2}}\left(2 a_{11} a_{13}-4 A_{2} a_{03}\right), d_{4}=\frac{1}{A_{2}^{2}}\left(a_{12}{ }^{2}-4 A_{2} a_{04}\right), \\
d_{5}=\frac{1}{A_{2}^{2}}\left(2 a_{13} a_{12}-4 A_{2} a_{05}\right), d_{6}=\frac{1}{A_{2}^{2}}\left(a_{13}{ }^{2}-4 A_{2} a_{06}\right) .
\end{gathered}
$$

By Lemma 2 we get

$$
\begin{aligned}
& \lim _{t \rightarrow 0}\left(4 d_{4} d_{6}-d_{5}^{2}\right)=0, \lim _{t \rightarrow 0}\left(3 d_{3} d_{4}-d_{2} d_{5}\right)=0, \\
& \lim _{t \rightarrow 0}\left(4 d_{4} d_{1}-d_{2}{ }^{2}\right)=0,
\end{aligned}
$$

thus, $\lim _{t \rightarrow 0} W(t, x, z)=0$. In the identity (13) taking

$$
\begin{aligned}
& y=\phi=-\frac{d_{5}}{2 d_{4}} z-\frac{d_{2}}{2 d_{4}} . \text { We obtain } \\
& W_{t}^{\prime}+W_{x}^{\prime} P(t, x, \phi)+W_{z}^{\prime} Q(t, x, \phi, z)= \\
& 2 W\left(\frac{A_{1}(t, x, \phi, z)}{A_{2}(t, x)} \mu_{3}(t, x)-\mu_{2}(t, x, \phi, z)\right) .
\end{aligned}
$$

By the uniqueness of solution of initial problem of linear partial differential equation, we get $W(t, x, z) \equiv 0$. Therefore

$$
\Delta=d_{4}\left(y+\frac{d_{5}}{2 d_{4}} z+\frac{d_{2}}{2 d_{4}}\right)^{2} .
$$

Using the relation (7), we obtain

$$
\begin{aligned}
F_{2} & =-\frac{a_{11}+a_{12} y+a_{13} z}{2 A_{2}}+\frac{1}{2} \sqrt{d_{4}}\left(y+\frac{d_{5}}{2 d_{4}} z+\frac{d_{2}}{2 d_{4}}\right) \\
& =f_{21}(t, x)+f_{22}(t, x) y+f_{23}(t, x) z .
\end{aligned}
$$

By the relation (6), we get

$$
F_{3}=\lambda_{1}+\lambda_{2} F_{2}=f_{31}(t, x)+f_{32}(t, x) y+f_{33}(t, x) z .
$$

$2^{*}$. If $C_{1} \neq 0$. From (10) follows $F_{2}=-\frac{C_{0}}{C_{1}}$. By the express of $A_{i}, B_{j}(i=0,1,2, j=0,1,2,3)$, we know that $C_{1}$ is a quadratic polynomial respect of $y, z, C_{0}$ is a cubic polynomial respect to $y, z$. Substituting $F_{2}=$ $-C_{0} / C_{1}$ into relation (7), we get $C_{1}\left(C_{0} A_{1}-C_{1} A_{0}\right)=$ $A_{2} C_{0}{ }^{2}$. It implies that $C_{1}$ is divided by $C_{0}$ or $A_{2}$, and $F_{2}=\sum_{i+j=0}^{3} f_{2 i j}(t, x) y^{i} z^{j}$, substituting it into (7) and equating the coefficients of like powers of $y$ and $z$ implies $f_{2 i j}(t, x)=0, i+j>1$. Thus,

$$
F_{i}=f_{i 1}(t, x)+f_{i 2}(t, x) y+f_{i 3}(t, x) z, i=2,3 .
$$


Summarizing the above, the proof is completed.

Obviously, from the relation (7) implies

Theorem 2. Let

$$
F_{1}=x, p_{i}(0, x)=0(i=1,2,3), A_{2}=0, A_{1} \neq 0 \text {. }
$$

Then

$$
\begin{aligned}
& F_{2}=-\frac{A_{0}}{A_{1}}=-\frac{a_{01}+a_{02} y+a_{03} z+a_{04} y^{2}+a_{05} y z+a_{06} z^{2}}{a_{11}+a_{12} y+a_{13} z}, \\
& F_{3}=\lambda_{1}+\lambda_{2} F_{2} .
\end{aligned}
$$

Case 2. $p_{3}(t, x) \equiv 0, p_{2} \neq 0$.

Applying identity (5) yields

$$
F_{2}=-\frac{p_{1}+\bar{p}_{1}}{\bar{p}_{2}}-\frac{p_{2}}{\bar{p}_{2}} y=\zeta_{1}+\zeta_{2} y,
$$

where $\zeta_{1}=-\frac{p_{1}+\bar{p}_{1}}{\bar{p}_{2}}, \zeta_{2}=-\frac{p_{2}}{\bar{p}_{2}}$.

Differentiating this identity respect to $t$ implies

$$
M_{0}+M_{1} F_{3}+M_{2} F_{3}^{2}=0,
$$

where

$$
\begin{aligned}
& M_{0}=D\left(\zeta_{1}+\zeta_{2} y\right)+\bar{q}_{1}+\bar{q}_{2} F_{2}+\bar{q}_{4} F_{3}^{2}= \\
& m_{01}+m_{02} y+m_{03} z+m_{04} y^{2}+m_{05} y z+m_{06} z^{2}, \\
& M_{1}=\bar{q}_{3}+\bar{q}_{5} F_{2}=m_{11}+m_{12} y, M_{2}=\bar{q}_{6}, \\
& m_{01}=\zeta_{1 t}^{\prime}+\zeta_{1 x}^{\prime} p_{1}+\zeta_{2} q_{1}+\bar{q}_{1}+\bar{q}_{2} \zeta_{1}+\bar{q}_{4} \zeta_{1}^{2} ; \\
& m_{02}=\zeta_{1 x}^{\prime} p_{2}+\zeta_{2 x}^{\prime} p_{1}+\zeta_{2 t}^{\prime}+\zeta_{2} q_{2}+\bar{q}_{2} \zeta_{2}+2 \bar{q}_{4} \zeta_{1} \zeta_{2} ; \\
& m_{04}=\zeta_{2 x}^{\prime} p_{2}+\zeta_{2} q_{4}+\bar{q}_{4} \zeta_{2}^{2} ; \\
& m_{03}=\zeta_{2} q_{3}, m_{05}=\zeta_{2} q_{5}, m_{06}=\zeta_{2} q_{6}, \\
& m_{11}=\bar{q}_{3}+\bar{q}_{5} \zeta_{1}, m_{12}=\bar{q}_{5} \zeta_{2} .
\end{aligned}
$$

Similarly, we obtain the following conclusion:

Lemma 3. Let $p_{3}=0, p_{2} \neq 0, q_{6} \neq 0, F_{1}=x$ and $\lim _{t \rightarrow 0} \frac{m_{0 i}}{q_{6}}(i=1,2, \ldots, 5)$ and $\lim _{t \rightarrow 0} \frac{p_{1}}{\bar{p}_{2}}$ exist. Then

$$
\begin{aligned}
& \lim _{t \rightarrow 0} \frac{m_{0 j}}{q_{6}}=0(j=1,2,4), \lim _{t \rightarrow 0} \frac{p_{1}+\bar{p}_{1}}{\bar{p}_{2}}=0, \lim _{t \rightarrow 0} \frac{p_{2}}{\bar{p}_{2}}=-1 \\
& \lim _{t \rightarrow 0} \frac{m_{03}+m_{11}}{q_{6}}=0, \lim _{t \rightarrow 0} \frac{m_{05}+m_{12}}{q_{6}}=0, \lim _{t \rightarrow 0} \frac{q_{6}}{\bar{q}_{6}}=-1 .
\end{aligned}
$$

Theorem 3. Let the conditions of Lemma 1 and Lemma 3 satisfy. Then

$$
\begin{aligned}
& F_{2}=\zeta_{1}(t, x)+\zeta_{2}(t, x) y, \\
& F_{3}=f_{31}(t, x)+f_{32}(t, x) y+f_{33}(t, x) z .
\end{aligned}
$$

Theorem 4. Let

$$
\begin{aligned}
& p_{i}(0, x)=0(i=1,2), q_{3}(t, x) \equiv 0, q_{6}(t, x) \equiv 0 \\
& m_{11}{ }^{2}+m_{12}{ }^{2} \neq 0, F_{1}=x
\end{aligned}
$$

$$
\begin{aligned}
& F_{2}=\zeta_{1}(t, x)+\zeta_{2}(t, x) y, \\
& F_{3}=-\frac{m_{01}+m_{02} y+m_{03} z+m_{04} y^{2}+m_{05} y z}{m_{11}+m_{12} y} .
\end{aligned}
$$

Theorem 5. For the system (3), if the following conditions satisfy

$$
\begin{aligned}
& p_{1}+\bar{p}_{1}+\bar{p}_{2} f_{21}+\bar{p}_{3} f_{31}=0, p_{2}+\bar{p}_{2} f_{22}+\bar{p}_{3} f_{32}=0 \text {, } \\
& p_{3}+\bar{p}_{2} f_{23}+\bar{p}_{3} f_{33}=0, f_{21}(0, x)=0, f_{31}(0, x)=0 \text {, } \\
& \left(\begin{array}{l}
f_{21 t}^{\prime} \\
f_{31 t}^{\prime}
\end{array}\right)+\left(\begin{array}{l}
f_{21 x}^{\prime} \\
f_{31 x}^{\prime}
\end{array}\right) p_{1}+\left(\begin{array}{l}
f_{22} \\
f_{23}
\end{array}\right) q_{1}+\left(\begin{array}{l}
f_{23} \\
f_{33}
\end{array}\right) r_{1}+\left(\begin{array}{l}
Q\left(-t, x, f_{21}, f_{31}\right) \\
R\left(-t, x, f_{21}, f_{31}\right)
\end{array}\right) \\
& =0 \text {, } \\
& \left(\begin{array}{ll}
f_{22 t}^{\prime} & f_{23 t}^{\prime} \\
f_{32 t}^{\prime} & f_{33 t}^{\prime}
\end{array}\right)+\left(\begin{array}{ll}
f_{22 x}^{\prime} & f_{23 x}^{\prime} \\
f_{32 x}^{\prime} & f_{33 x}^{\prime}
\end{array}\right) p_{1}+\left(\begin{array}{ll}
f_{21 x}^{\prime} p_{2} & f_{21 x}^{\prime} p_{3} \\
f_{31 x}^{\prime} p_{2} & f_{32 x}^{\prime} p_{3}
\end{array}\right)+ \\
& \left(\begin{array}{ll}
f_{22} & f_{23} \\
f_{32} & f_{33}
\end{array}\right)\left(\begin{array}{ll}
q_{2} & q_{3} \\
r_{2} & r_{3}
\end{array}\right)+\left(\begin{array}{ll}
\bar{Q}_{y}^{\prime} & \bar{Q}_{z}^{\prime} \\
\bar{R}_{y}^{\prime} & \bar{R}_{z}^{\prime}
\end{array}\right)_{(t, x, 0,0)}=0, \\
& \left(\begin{array}{ll}
f_{22 x}^{\prime} p_{2} & f_{23 x}^{\prime} p_{3} \\
f_{32 x}^{\prime} p_{2} & f_{33 x}^{\prime} p_{3}
\end{array}\right)+\left(\begin{array}{ll}
f_{22} & f_{23} \\
f_{32} & f_{33}
\end{array}\right)\left(\begin{array}{ll}
q_{4} & q_{6} \\
r_{4} & r_{6}
\end{array}\right)+ \\
& \left(\begin{array}{ll}
\bar{q}_{4} & \bar{q}_{6} \\
\bar{r}_{4} & \bar{r}_{6}
\end{array}\right)\left(\begin{array}{ll}
f_{22}^{2} & f_{23}^{2} \\
f_{32}^{2} & f_{33}^{2}
\end{array}\right)+\left(\begin{array}{ll}
f_{22} f_{23} \bar{q}_{5} & f_{23} f_{33} \bar{q}_{5} \\
f_{22} f_{23} \bar{r}_{5} & f_{23} f_{33} \bar{r}_{5}
\end{array}\right)=0, \\
& \left(\begin{array}{ll}
f_{22 x}^{\prime} & f_{23 x}^{\prime} \\
f_{32 x}^{\prime} & f_{33 x}^{\prime}
\end{array}\right)\left(\begin{array}{l}
p_{3} \\
p_{2}
\end{array}\right)+\left(\begin{array}{ll}
f_{22} & f_{23} \\
f_{32} & f_{33}
\end{array}\right)\left(\begin{array}{l}
q_{5} \\
r_{5}
\end{array}\right)+ \\
& \left(\begin{array}{c}
2 \bar{q}_{4} f_{22} f_{23}+\bar{q}_{5}\left(f_{22} f_{33}+f_{23} f_{32}\right)+2 \bar{q}_{6} f_{32} f_{33} \\
2 \bar{r}_{4} f_{22} f_{23}+\bar{r}_{5}\left(f_{22} f_{33}+f_{23} f_{32}\right)+2 \bar{r}_{6} f_{32} f_{33}
\end{array}\right)=0, \\
& \left(\begin{array}{ll}
f_{22} & f_{23} \\
f_{32} & f_{33}
\end{array}\right)\left(\begin{array}{ll}
\bar{f}_{22} & \bar{f}_{23} \\
\bar{f}_{32} & \bar{f}_{33}
\end{array}\right)=\left(\begin{array}{ll}
f_{22}(0, x) & f_{23}(0, x) \\
f_{32}(0, x) & f_{33}(0, x)
\end{array}\right)=\left(\begin{array}{ll}
1 & 0 \\
0 & 1
\end{array}\right) .
\end{aligned}
$$

Then $F=\left(\begin{array}{c}x \\ f_{21}+f_{22} y+f_{22} z \\ f_{31}+f_{32} y+f_{33} z\end{array}\right)$ is the RF of system (3).

Besides this, if the system (3) is $2 \omega$--periodic with respect to $t$, then its solution $(x(t), y(t), z(t))$ defined on the interval $[-\omega, \omega]$ with initial condition $(x(-\omega)$, $y(-\omega), z(-\omega))=\chi$ is $2 \omega$--periodic if and only if $F(-\omega, \chi)=\chi$.

Proof. By checkout of the BR it is proved that the function $F=\left(x, f_{21}+f_{22} y+f_{23} z, f_{31}+f_{32} y+f_{33} z\right)^{T}$ is the RF of system (3). At this moment, the Poincare mapping of periodic system (3) is $T(x, y, z)=F(-\omega, x, y$, $z)$. By the previous introduction the assertions of the present theorem is hold. The proof is finished.

Under the hypotheses of Theorem 5, the first component of solution of system (3) is even function.

Example: Differential system 


$$
\left\{\begin{array}{l}
x^{\prime}=\left(1-e^{\sin t}\left(1-x^{3} \sin t\right)\right) y-x^{2} z \sin t \\
y^{\prime}=\frac{1}{2} y \cos t\left(x^{6} \sin ^{2} t+x^{3}-1\right)-\frac{1}{2} z x^{2} \cos t e^{-\sin t}\left(1+\sin t+x^{3} \sin ^{2} t\right) \\
+y^{2}\left(3 x^{2} \sin t-x^{5} \sin ^{2} t\right)+y z e^{-\sin t}\left(x^{4} \sin ^{2} t-2 x \sin t\right) \\
z^{\prime}=\frac{1}{2} y x^{4} \cos t e^{\sin t}\left(1-\sin t+x^{3} \sin ^{2} t\right)+\frac{1}{2} z \cos t\left(1-x^{3}-x^{6} \sin ^{2} t\right) \\
-y^{2}\left(x^{6} \sin ^{2} t-4 x^{3} \sin t\right) e^{\sin t}-y z\left(3 x^{2} \sin t-x^{5} \sin ^{2} t\right)
\end{array}\right.
$$

has RF

$$
F(t, x, y, z)=\left(\begin{array}{c}
x \\
e^{\sin t}\left(1-x^{3} \sin t\right) y+x^{2} z \sin t \\
-x^{4} y \sin t+e^{-\sin t}\left(1+x^{3} \sin t\right) z
\end{array}\right) .
$$

Since this system is a $2 \pi$--periodic system, and $F(-\pi, x, y, z) \equiv(x, y, z)^{T}$, by Theorem 5 , all the solutions of the considered system defined $[-\pi, \pi]$ are $2 \pi$--periodic.

\section{References}

[1] V. I. Arnold, "Ordinary Differential Equation," Science Press, Moscow, 1971, pp. 198-240.
[2] V. I. Mironenko, "Reflective Function and Periodic Solution of the Differential System," University Press, Minsk, 1986, pp. 12-26.

[3] V. I. Mironenko, "The Reflecting Function and Integral Manifolds of Differential Systems," Differential Equations, Vol. 28, No. 6, 1992, pp. 984-991.

[4] V. V. Mironenko, "Time Symmetry Preserving Perturbations of Differential Systems," Differential Equations, Vol. 40, No. 20, 2004, pp. 1395-1403.

[5] P. P. Verecovich, "Nonautonomous Second Order Quadric Wystem Equivalent to Linear System," Differential Equations, Vol. 34, No. 12, 1998, pp. 2257-2259.

[6] E. V. Musafirov, "Differential Systems, the Mapping over Period for Which is Represented by a Product of Three Exponential Matrixes," Journal of Mathematical Analysis and Applications, Vol. 329, No.1, 2007, pp. 647-654.

[7] Z. X. Zhou, "On the Reflective Function of Polynomial Differential System," Journal of Mathematical Analysis and Applications, Vol. 278, No. 1, 2003, pp. 18-26.

[8] Z. X. Zhou and Y. X. Yan, "Reflecting Function and Periodic Solution of Quadratic Differential System," Applied Mathematics, Vol. 15, No. 4, 2002, pp. 85-91. 